\title{
Construction of Prevention and Control Mechanism for the Elderly Against the Background of COVID- 19
}

\author{
Bing $\mathrm{Li}^{1, *}$ Yushi Luo ${ }^{1}$ \\ ${ }^{1}$ School of Public Affairs and Administration, UESTC, Chengdu, Sichuan, China \\ *Corresponding author. Email: 1065810507@qq.com
}

\begin{abstract}
Elderly people are prone to infection in public health events due to their weakened physical function and weakened immunity. The outbreak of COVID-19 has promoted the improvement of the prevention and control mechanism of the elderly population and the modernization of the governance system and governance capacity. Based on the theory of public crisis management, this study elaborates the construction of the mechanism of prevention and control management of the elderly population from the aspects of crisis reduction management, crisis preparation management, crisis response management and crisis recovery management, so as to improve the prevention and control mechanism of major epidemic and the national public health emergency management system.
\end{abstract}

Keywords: Public crisis management, Elderly population, Prevention and control mechanism, COVID-19.

\section{INTRODUCTION}

The sudden outbreak of COVID-19 in early 2020 triggered a global health crisis. At the same time, the elderly are vulnerable to COVID-19 infection due to their own weakened immune system, weak resistance and common underlying chronic diseases. According to the Influences of COVID19 on the Elderly released by UN Secretary-General Antonio Guterres on May 1, 2020, "the mortality rate of the elderly infected with novel coronavirus is higher, and the mortality rate of the elderly over 80 years old is as high as five times of the average" [1]. As of September 11, 2020, people over 65 years old accounted for only 12 percent of all confirmed cases of infection, but 66 percent of deaths, according to United Nations population officials who analyzed the situation in 55 countries, including the United States. People under 44 years of age accounts for $60 \%$ of confirmed cases, and only $7 \%$ of deaths. [2] In addition to its health

*Fund: Key Research Base of Humanities and Social Sciences of Sichuan Education Department - Southwest Medical University "Sichuan Hospital Management and Development Research Center", (Key funded Project SCYG2019-02) "Research on the Optimization of Community-Based Model of Elderly Nursing Care Combined with Medical Care Service". risks, COVID-19 puts older people at risk of poverty, discrimination and isolation, and the digital divide, bringing incalculable fear and suffering to the elderly population [1].

On March 11, 2020, the World Health Organization (WHO) declared COVID-19 a "global pandemic" [3]. Professor Zhang Wenhong said in the 13th Symposium on High-end Medical Service and Construction that COVID-19 has become a "resident virus" [4]. The sudden and long-term COVID-19 outbreak has posed a huge challenge to China's governance capacity and governance system, and opened a "window of opportunity" for China's security risk prevention and control and emergency management mechanism. On February 14, 2020, General Secretary Xi presided over the 12th meeting of the Commission for Deepening Overall Reform of the CPC Central Committee, stressing that "in view of the shortcomings and deficiencies exposed by the epidemic, we should strengthen the weak links, plug the loopholes and strengthen the weak points", "improve the prevention and control system of major epidemic and improve the national public health emergency management system" [5]. Therefore, based on the $4 \mathrm{R}$ theory of public crisis management, 
combined with the experience of fighting against COVID-19 and aiming at the characteristics of the elderly population, this study constructed the normal and abnormal management mechanism for prevention and control of the elderly population (two-state mechanism). Efforts were made to deepen the reform of emergency management, optimize the public health emergency management system and enhance the emergency management capacity, and enhance its ability to withstand risks.

\section{PREVENTION AND CONTROL CHARACTERISTICS OF ELDERLY POPULATION IN THE CONTEXT OF COVID-19}

\subsection{Decline in Physical Function Makes the Elderly Vulnerable to Health Shocks}

The physical indicators of the elderly become worse with the increase of age. They often suffer from basic diseases and chronic diseases, and their immune function is weak. They are susceptible to novel coronavirus infection, and once infected, complications are likely to occur, leading to health deterioration, exacerbation of the disease and even death. At the same time, the isolation policy was adopted in various places during the epidemic. As a result, the elderly were obstructed in social interaction and information occlusion, which could easily lead to negative emotions such as loneliness, anxiety and depression, and induce psychological diseases.

\subsection{Inadequate Digital Capabilities and Limited Scientific Protection Have Hindered the Implementation of Prevention and Control Measures}

Affected by subjective and objective factors such as economic conditions, educational level, living environment, scientific and technological development, and physical health, some elderly people lack digital ability. They are limited in the ability of information acquisition and screening [6], unable to effectively absorb correct knowledge and information and experience to carry out self-scientific protection, and may also absorb wrong information and fall into the trap of fraud. They have a low degree of acceptance, mastery and use of new technologies and new things, and are unable to use smart devices smoothly. They are trapped in smart technologies required for epidemic prevention, such as health codes and online clocking reports, and fall into the digital divide, which to some extent affects the implementation of prevention and control policies. At the same time, it is difficult for them to adapt to the intelligent communication mode in the information age, which will lead to the lack of discourse power of the elderly group and difficulty in expressing their demands.

Vulnerable physical and psychological conditions lead to the elderly's dependence on action support and medical support [6] First of all, aging leads to the deterioration of the physical function of the elderly, and in severe cases, the loss of self-care ability. At the same time, the elderly with underlying, chronic and other medical conditions need to maintain basic and appropriate medical behavior during isolation. Secondly, the elderly, especially those who are widows, living alone and disabled, will have a sense of loss of value and emotion. The isolation policy will block the channels for the elderly to participate in the society, weaken social activities and aggravate psychological pressure. Medical care, health monitoring and care services are conducive to improving the physical function of the elderly, restoring their self-care ability, maintaining physical health and relieving psychological pressure. No matter at home, in the community or in institutions, the elderly have a considerable demand for action support and medical support. Finally, the isolation policy makes life inconvenient. During the period of home isolation, the purchase of materials is inconvenient, and the demands of normal life, such as food, medicine and daily necessities, shall be met.

\subsection{Establishing a Two-state Epidemic Prevention and Control Mechanism for the Elderly}

\subsubsection{Improving Legal Norms,}

Strengthening Training and Exercises, Strengthening Capacity Upgrading, and

Building a Crisis Reduction Management Mechanism

Crisis reduction management is the core of crisis management, which starts from the environment, structure, system and personnel to formulate crisis reduction strategies and reduce the occurrence and impact of crisis [7]. China's documents on prevention and control and social assistance mostly emphasize the disadvantaged groups, and pay little attention to the heterogeneity of the groups, let alone the lack of policy support measures specifically aimed at the elderly groups [8]. At the same time, the elderly themselves are also faced with information dilemma, 
knowledge dilemma, digital dilemma, discourse power dilemma, etc. Therefore, in terms of crisis reduction, the crisis management and relief measures for the elderly should be improved. The transfer and integration of personnel should be guaranteed, and the continuous relief policy should be implemented to ensure that "assistance is available and timely" for the elderly [8]. Secondly, the emergency capacity building of the elderly should be paid attention to. Regular science lectures, training exercises and other activities are carried out to improve the emergency knowledge and quality of the elderly. Concentrated teaching and learning of intelligent technology equipment will be conducted to accelerate the "electric shock" of the elderly, improve their information technology ability and strengthen their demand expression ability. Finally, specialized personnel should be brought in to strengthen the drill and training of crisis events, enhance the crisis analysis ability of relevant personnel, quickly identify crisis events and vulnerable groups, and improve the emergency command ability.

\subsubsection{Improving the Early Warning System, Activating the Information and Resource Systems, and Establishing a Crisis Preparation and Management Mechanism}

Crisis preparation management corresponds to prior management, mainly through early warning and monitoring of abnormal environmental signals, to make quick and necessary response and improve the ability of crisis prevention [7]. The early warning mechanism of emergency events should be improved, corresponding early warning institutions such as crisis command center should be established, public health event prevention teams should be set up, all kinds of health safety information should be collected and abnormal information should be analyzed, so as to enhance the crisis early warning ability. The construction of emergency material support system should be paid attention to. The principles of "centralized management, unified allocation, peacetime service, emergency response in case of disaster, combination of production and storage, economy and efficiency" should be followed to improve the relevant working mechanism and emergency plan [5]. The normalization of information resource guarantee system should also be valued. Information integration and sharing should be promoted with the community as the main body and the civil affairs and social charity departments as the auxiliary. The community integrates the information of the elderly in the community and carries out normal management. The community integrates the information of the elderly in the community and carries out normal management. During the abnormal emergency period, information should be shared. All service subjects provide convenient epidemic prevention services by using cloud services of information technology to timely and accurately grasp epidemic prevention information and situation. For example, health monitoring, safety warning, remote service and rescue should be carried out on the initiative of the elderly according to their electronic health records.

\subsubsection{Exploring a Linkage Mechanism, Improving the Medical System, and Building a Crisis Response Management Mechanism}

Crisis preparation management corresponds to management in the process, emphasizing the handling means and coping strategies adopted when a crisis occurs, and improving crisis response ability through communication, coordination, planning and decision-making, dynamic adjustment and other positive measures to deal with crisis events [7]. The linkage mechanism between government departments and enterprises, social organizations and other nongovernmental organizations should also be explored, so as to transform the linkage loss in the abnormal period into the running in the normal period, and incorporate the abnormal emergency response capacity building into the normal cooperative action. This will improve China's ability to respond to health risks for the elderly at a particular time. The hierarchical diagnosis and treatment system should be actively promoted and medical resources should be reasonably allocated [9]. The elderly should be guided to seek first diagnosis at the grassroots level, two-way referral and triage should be strengthened, remote diagnosis and treatment should be implemented, and the effectiveness and efficiency of medical management in special times should be improved, and the health management of the elderly in non-crisis times should not be omitted or ignored [10]. The legal system should leave room for flexibility. Unconventional sudden crisis events involve multi-subject connection of different levels, different fields and different regions in the process of pre-plan initiation, rescue operation and situation control. At the same time that the legal norms of public health emergencies are perfected, the subject coordination, resource allocation and power attribution in special period should be more flexible, so as to enhance the response capacity and resilience 
of organizations and ensure the dynamic and controllable emergency management.

\subsubsection{Improving the Capacity for Follow-up Recovery, Attaching Importance to the Summary of Experience and Lessons, and Building a Crisis Recovery Management Mechanism}

Crisis recovery management corresponds to postcrisis management. First, the recovery and construction of production and life after the crisis; second, the experience of crisis response is summarized. Through reviewing the whole crisis response process, the experience and lessons are summarized and the existing mechanism is improved and optimized to better cope with the public crisis that may be encountered in the future. The first is to establish the psychological intervention mechanism of the elderly group. Due to age, physiology, pathology and environment, the elderly group is more vulnerable than other groups, so it is necessary to carry out post-crisis psychological recovery construction for the elderly group to help them recover to normal life. The second is to speed up the restoration of the normal operation of medical institutions to ensure the normal provision of medical services. The number of patients should be limited to prevent the recurrence of the epidemic. The third is to sort out and analyze the experience and deficiencies of the previous epidemic prevention and control work, consolidate the achievements of crisis management, and improve the public security risk prevention and control and emergency management mechanism.

\subsubsection{Strengthening the Application of Science and Technology to the Market and Giving Full Play to Their Effectiveness}

The application of science and technology should be strengthened to enhance ability to apply new information technologies such as big data and artificial intelligence. Big data, information and communication technology, artificial intelligence and other technologies are employed to establish and improve the decision-making assistance system for the emergency management of the epidemic for the elderly, as well as the technical support system for the whole process of detection and treatment for the elderly. In terms of risk prediction, the big data model of the elderly population should be used as the basis for risk prediction and assessment of the health status, mental state and infection rate of the elderly population in a specific area from physiological, psychological and pathological aspects, so as to monitor and warn potential, hidden and changing risk problems among the elderly population. As for epidemic prevention and control, the advantages of financial media should be fully utilized, cloud technology and information technology should be used to smooth the information channels of the elderly, and the prevention and control publicity should be strengthened to ensure that the epidemic prevention and control information they receive is true and effective, so as to effectively improve their awareness of prevention and self-protection ability. As for epidemic care, artificial intelligence technologies such as remote diagnosis and treatment and virtual monitoring should be fully adopted to overcome epidemic barriers, provide remote medical services for the elderly and promote rational allocation of medical resources.

\section{CONCLUSION}

Despite the difficulties and challenges it has brought to social governance, the COVID-19 epidemic has also created opportunities for China to improve the mechanism of cooperation among various parties in social governance and the mechanism of crisis response and management. The reduction of the impact of the crisis and the improvement of the ability to prevent and respond to the crisis are conducive to subsequent recovery, and the application of science and technology is strengthened. The crisis management mechanism has been established to better deal with emergencies and promote the modernization of social governance.

Based on the experience of prevention and control of COVID-19, this study analyzed the characteristics of middle-aged and elderly groups in the prevention and control of COVID-19, and proposed to construct a two-state mechanism for prevention and control of COVID-19 for the elderly from the perspective of public crisis management. To some extent, this is conducive to promoting the modernization of national governance capacity and governance system and enriching the research results of social governance in the post-epidemic era. However, this study still has the following shortcomings. Firstly, there is relatively insufficient theoretical support. Secondly, the constructed mechanism still needs further empirical test to prove its universality and operability. In view of the above deficiencies, this study will continue to monitor the progress of global epidemic prevention 
and control, and at the same time establish close contact with grassroots governance personnel.

\section{AUTHORS' CONTRIBUTIONS}

Bing $\mathrm{Li}$ is responsible for the Research design, as well as the review and revision of the articles. Yushi Luo contributed to method design and article writing.

\section{REFERENCES}

[1] Talengen Care. Secretary-General of the United Nations released "The Impact of the New Crown Epidemic on the Elderly" [EB/OL]. (in Chinese)

https://www.sohu.com/a/393390346_12012460 5.

[2] The data show that the death rate of the elderly from the epidemic far exceeds that of the young [EB/OL]. (in Chinese) https://baijiahao.baidu.com/s?id=168040071293 4023321\&wfr=spider \&for=pc,2020-10-12.

[3] WHO declared the new crown pneumonia a global pandemic, with the full text of the speech! [EB/OL]. (in Chinese) https://www.163.com/dy/article/F7HK5OSR051 1VCHN.html, 2020-3-12.

[4] Zhang Wenhong's latest voice: The new crown has become a resident virus in the world and hopes to achieve widespread vaccination as soon as possible [EB/OL]. (in Chinese) https://baijiahao.baidu.com/s?id=169990848513 $7464881 \& w f r=$ spider $\&$ for $=p c, 2020-5-16$.

[5] Xi Jinping presided over the twelfth meeting of the Central Comprehensive Deepening Reform Committee and emphasized: perfecting major epidemic prevention and control systems and mechanisms, and perfecting the national public health emergency management system [EB/OL]. (in Chinese) http://www.gov.cn /xinwen/2020-02/14/content_5478896.htm, 2020-2-14.

[6] Liu Yuan. Vulnerability Analysis of the Rights of the Elderly in Public Health Emergencies Against the Background of COVID-19 [J]. Human Rights, 2020(03): 87-102. (in Chinese)

[7] Yan Deru; Ma Yueqi. The Establishment of the Whole-Process Community Emergency Information Management System:An Analysis Based on 4R Crisis Management Theory [J].
The Northern Forum, 2021, No. 285, 78-87. (in Chinese)

[8] Ma Guangbo; Ni Peifan. Research on "Emergency Rescue Difficulty" of Lonely Elderly People Based on Community Public Health Emergency Management [J]. Journal of Yibin University, 2021, 21(03): 48-55. (in Chinese)

[9] Promote the popularization of high-quality medical resources and advance the equalization of basic medical and health services [EB/OL]. (in Chinese) http://www.gov.cn/zhengce/201509/01/content_2923441.htm?from=timeline\&isa ppinstalled $=0$, 2015-9-1.

[10] Feng Qilong. Human Health Community: The Value Orientation of the Development of Regional Medical Association in Yangtze River Delta [J]. Nanjing Journal of Social Sciences, 2021, (05): 63-70. (in Chinese) 